\title{
TERCERA MESA REDONDA
}

La primera pregunta va dirigida al doctor Murray: - ¿Hay diferencia cuanti o cualitativa entre las lesiones vesicales, cuando el radium se aplica en una sola tanda o sesión, o cuando se aplica en dos sesiones con un intervalo suficiente de descanso?

Doctor Murray: —No, en realidad no hay diferencia. Dependen las lesiones más de la dosis total que de la dosis fraccionada.

También para el doctor Murray, otra pregunta: - ¿Es ventajosa la irradiación previa de Alberheim para el tratamiento del $\mathrm{Ca}$ uterino? Está un poco fuera de lo que se discute en la Mesa Redonda pero no tenemos inconveniente en que el doctor Murray nos dé su opinión.

Doctor Murray: -Al respecto yo me referí a la irradiación previa no en el carcinoma del cuello, sino en el carcinoma del endometrio, si se tiene en cuenta que no la hacemos nunca en el carcinoma del cuello, salvo en los casos en que está indicado.

Para el doctor Rogelio Arias de Panamá: -Resultados de los ejercicios esfinterianos para el tratamiento de la incontinencia urinaria leve.

Doctor Arias: - Los resultados de los ejercicios perineales en el tratamiento de la incontinencia urinaria leve son buenos, diría que en menos del $25 \%$ de los casos. Pero ayudándolos, sobre todo en la mujer menopáusica, con estrógenos, muchas veces son satisfactorios y así se elimina una operación innecesaria.

Doctor Jordán: - Hay una pregunta dirigida a la Mesa en general y que me tomo la libertad de contestar:

-Agradecemos una breve explicación de la succión extra-peritoneal, con sonda de goma, según la técnica de Simeon Pratt de la Mayo-Clinic.

- Hay varias formas de hacerla: Una forma extra-peritoneal por catéteres que se abocan a la piel.

Se puede hacer también con una sonda en $T$, colocada en vagina. Se pone un aparato de succión continua y en esta forma hemos obtenido en el Instituto Nacional de Cancerología, algo similar a lo publicado por los autores en un promedio de trescientos a trescientos cincuenta cmts. cúbicos de líquido sero-sanguinolento en los primeros dos o tres días posteriores a la intervención. Pensamos que esto disminuye la formación de tejido fibroso y la formación de quistes peritoneales, como se ha reportado en este tipo de intervenciones quirúrgicas. La vía vaginal probablemente sea más fácil para 
utilizar, pero la vía por la piel da también excelentes resultados.

A la Mesa: - ¿Aconseja rutinizar la urografía pre-operatoria, como contribución a la profilaxis de las fístulas post-operatorias en casos de tumores parauterinos y en los fijos o en los enclavados en la pelvis?

Doctor Aparicio: —En relación con el problema de si se debe o no hacer una radiografía previa a cada intervención cuando se trata de tumores pélvicos grandes, estoy absolutamente convencido de que en los medios quirúrgicos hospitalarios se debe siempre practicar. Ustedes recuerdan las variantes anatómicas del uréter en este tipo de intervenciones; entonces con una radiografía previa simple, una urografía, se puede por lo menos tener en mente el problema del uréter para ponerle muchísima atención en cualquier momento de la intervención. De manera que mientras sea posible, aconsejo que se haga siempre previamente a la intervención.

Para el doctor Bussamara Neme del Brasil la siguiente pregunta dividida en dos partes: A) — ¿Durante cuánto tiempo aconseja usted seguir los ejercicios perineales, antes de decidirse a operar?

B) - ¿Qué porcentaje de curaciones obtiene con la aplicación de los ejercicios perineales?

Doctor Neme: —Los ejercicios perineales y la intervención quirúrgica no tienen la misma finalidad curativa. Estos contribuyen a la terapéutica. Es verdad que como profilaxis se recomienda a todas las mujeres en el puerperio reciente y tardío, que hagan los ejercicios, para tornar menos intenso el proceso de incontinencia y reducir la intervención a la necesidad de aquella mujer que tenga un número de hijos que no desee aumentar. Ahora, es una utopía el que con estos ejercicios se cure una incontinencia que viene durando después de tres o cuatro meses. Realmente, yo no podría responder cuál es la incidencia de las curaciones de este tipo. Pero sí recomiendo a todos los obstetras que en su asistencia aconsejen a las puérperas que hagan los ejercicios perineales.

La siguiente pregunta al doctor Delfino Gallo: — ¿Cuáles son sus resultados y casuística en las intervenciones propuestas en incontinencia urinaria, y grandes fístulas vésico-urétero-vaginales?

Doctor Gallo: —El uso del asa sigmoide lo he empleado unas siete veces, y el doctor Longoria, de la Ciudad de México, lo ha usado, creo, en tres casos más. Este es a mi parecer el método más seguro de todos los que he descrito, por la circunstancia que ya había explicado de que el asa sigmoide está cubriendo el peritoneo; de tal manera que en unas cuantas horas, tal vez, o cuando menos en unos cuantos días se establecen sinequias entre la superficie cruenta que queda después de haber quitado la mucosa vaginal y el sigmoide. Además, viniendo la vascularización de un territorio vascular muy lejano, no hay el peligro de que surja por la esclerosis, dificultades en la irrigación; las arterias cólicas obviamente están perfectamente sanas y entonces se establece pronto la circulación. 
Realmente no he tenido ningún caso en que haya habido fracaso de la cura de la fístula. Lo que sí no es lo mismo es en que se haya obtenido continencia. Algunos de estos casos han permanecido incontinentes y han sido necesarias intervenciones del tipo retropúbico, suspensiones y en algunos casos no se ha logrado una continencia muy perfecta. El doctor Longoria me informó que ha tenido buenos resultados en las operaciones que ha practicado, pero realmente no conozco bien sus casos. En lo que se refiere a la interposición del útero despojado del cérvix y del endometrio, la he practicado también en muy pocos casos; nada más son seis casos hasta la fecha, con resultados bastante aceptables.

En lo que se refiere al uso del apéndice, fue iniciado hace aproximadamente siete años, fue publicado en un resumen en la Revista de Ginecología y Obstetricia de México, "sobre diez años de progreso en Urología ginecológica en México", y en un caso no se obtuvo ningún buen resultado, se necrosó el apéndice; en otro caso hubo estenosis; otro caso se perdió y los demás se han seguido y han salido bastante bien.

Hay otra pregunta para el doctor Gallo y es referente a: - El porcentaje de peritonitis que han tenido sus enfermas con este tipo de intervención.

Doctor Gallo: -En la intervención para usar el asa sigmoide, tuve un caso en que hubo oclusión; es decir, no se restableció bien el tránsito digestivo porque se produjo edema en el lugar de la anastomosis; esto obligó a hacer una colostomía en e traverso y se quitó el edema, no hubo necesidad de modificar para nada la anastomosis y posteriormente se hizo la cura de la colostomía. Este es el único caso de complicación; realmente con el uso de los antibióticos, usando bastante Neomicina, una buena dieta, pues la resección del asa sigmoide es una operación bastante simple, que no tiene complicaciones, haciéndola bien.

Para el doctor Néstor Velásquez: - Relación de la menopausia sobre la incontinencia de esfuerzo y acción de la estrogenoterapia en estos casos.

Doctor Velásquez: —Esa pregunta, bueno, ya quedó contestada después de escuchar a los relatores. De todas maneras nosotros practicamos en las enfermas añosas la citología vaginal, la cual nos da entonces el grado de estrogenismo y de acuerdo con ello empleamos el Estrógeno, el Compremín o cualquiera otro similar. Hemos tenido éxito en todos estos casos de incontinencia discreta, o de grado I, como la describen acá.

Hay una pregunta que originalmente iba dirigida al doctor Bedoya, pero que trasladamos al doctor Alvaro Fonnegra Miramón: —¿Qué sonda utilizan ustedes en la uretra para el post-operatorio?

Doctor Fonnegra: -Como se sabe, sistemáticamente se usa la sonda de Foley. Antiguamente se usaba la sonda de Petzer, pero ésta traumatiza mucho la uretra al retirarla; de tal manera que actualmente se usa siempre la sonda de Foley. 
A cualquier miembro de la Mesa que desee contestarla: -Experiencia en la cisto-uretrografía en contraste, utilizando la Cadenita de Bola y conclusiones.

Doctor Velázquez: En el trabajo que ustedes me escucharon, tengo reunidos 24 casos en los últimos tres años, en que he empleado este método, que fue propuesto anteriormente por Hopkins, me parece, y empleamos una cadenita de unos veinticinco centímetros, que introdujimos en la vejiga utilizando un uretroscopio, la camisa de un uretroscopio. En ios veinticuatro casos hemos observado, como ya vieron ustedes en las radiografías que expusimos, descenso de la base de la vejiga, en forma de embudo, y lo que es más importante, la pérdida del ángulo uretro-vesical en la radiografía oblicua.

Doctor Bedoya: -Pido perdón a la Mesa por haber llegado un poco tarde. En primer lugar en respuesta a la pregunta sobre qué sonda usamos en el post-operatorio, ya lo ha dicho el doctor Fonnegra, usamos la Foley.

En segundo lugar, respecto a esta pregunta, nosotros estamos usando desde el primer momento la cadena, con muy buenos resultados. Esto nos ha permitido hacer y seguir perfectamente bien todas las alteraciones que se suceden en el ángulo vésico-uretral. Lástima que no haya podido traer algunas diapositivas, para hacer alguna demostración práctica sobre la gran utilidad que tiene la cadena y que en todas nuestras enfermas ha sido perfectamente soportada, permitiéndonos a la larga seguir un post-operatorio también.

Doctor Arias: —En el problema del uso de la cadena, hay que tener en cuenta que como es un cuerpo extraño, que está en la base de la vejiga, la estimula algunas veces, y esta se contrae. Muchas de las películas que se muestran no son la forma como la vejiga va a actuar si no tuviera esa irritación de cuerpo extraño.

Una pregunta para el doctor Gallo _ ¿Cuánto tiempo permanece el "clamp" colocado, en su técnica, y en qué momento se retira?

Doctor Gallo: - Siempre pretendo dejar el "clamp" alrededor de dos semanas, pero en general molesta tanto a las mujeres, que no se les puede dejar más de diez o doce días. Teóricamente se debería dejar a lo menos quince días, pero por ser extremadamente molesto se tiene que retirar unos días antes de lo deseable. 